\title{
ASSESSMENT OF ANGIOSPERM PLANT DIVERSITY OF NIJHUM DWEEP, BANGLADESH
}

\author{
MOHAMMAD ZASHIM UDDIN ${ }^{1}$, MD. GOLAM KIBRIA AND MD. ABUL HASSAN \\ Department of Botany, University of Dhaka, Dhaka-1000, Bangladesh.
}

\begin{abstract}
The present article focuses the status of angiosperm plant diversity of Nijhum Dweep, a small Island in the Bay of Bengal close to Hatiya channel. From the analysis of the data a total of 152 plant species belonging to 56 families has been recorded. Among the recorded species, tree is represented by 66 , shrub by 15 , herbs by 58 and 13 by climbers. Of the species recorded from the area $51 \%$ species represented by 11 families and $49 \%$ represented by 45 families. Fabaceae appears to be largest in the Dicotyledones having 10 species whereas Poaceae is the largest in Monocotyledones having 12 species. Analysis confirmed that $68 \%$ of the recorded species found to be medicinal and $32 \%$ are used for other than medicinal purposes. Data analysis also showed that homesteads supported maximum plants followed by road side, cultivated land, mangrove and mangrove meadows. Collected data revealed that the occurrence of seven species namely Bruguiera gymnorrhiza, Diospyros blancoi, Derris trifoliata, Heliotropium curassavicum, Tamarix gallica, Typha elephentanea and Sarcolobus carinatus in the study area might be rare. Dolichandrone spathacea, a threatened of plant species of Bangladesh, was also found in this mangrove forest area. Through observations and discussion with local people, a number of threats to plant diversity have been identified. Finally, a number of possible conservation measures have been suggested for the management of angiosperm plant diversity of Nijhum Dweep.
\end{abstract}

Key words: Assessment, Angiosperm, Plant diversity, Nijhum Dweep

\section{Introduction}

Nijhum Dweep (Nijhum Island), is a tiny offshore island in the Bay of Bengal located between $21^{\circ} 35^{\prime} 0^{\prime \prime} \mathrm{N}$ and $92^{\circ} 01^{\prime} 0^{\prime \prime} \mathrm{E}$ in the southern part of Hatiya Upazila separated by Hatiya channel under Noakhali district. It is a cluster of several small accreditations mainly Char Osman, Char Kamla, Char Muri and Ballar Char. A virgin island with intertidal mudflats and sandflats has a scenic treasure trove having $20 \mathrm{kms}$ long sandy and grassy beach. Total area of the island is about 40390 ha.. In the year 1974, forest department started forestation program using the species of Sonneratia apetala (Keora) and Avicennia alba (Baine) in the northern part of the island. The forest area is about 9000 acres and is very dense with many other associated species. The forest bed is muddy and inundated by tidal actions twice in a day. The island is also dissected by small creeks

\footnotetext{
${ }^{1}$ Corresponding author: E-mail: zashim@du.ac.bd
} 
or canals and its centre part is under cultivation and human habitation. Generally, walk in the forest sometimes is not very easy because of channels and the presence of pneumatophore produced by Sonneratia apetala (Keora) and Avicennia alba (Baine) trees. The soil is highly alkaline. In the year of 2001, People's Republic of Bangladesh government has declared 16352.23 ha of forest land of Nijhum Dweep as a National Park for the protection of biodiversity. After that many media and enthusiastic people have highlighted the island for ecotourists to visit. According to local people and foresters reports, a good number of people has been visiting the island in winter taking much trouble in the journey. The area enjoys a moist tropical maritime climate and rainfall is frequent and heavy during the monsoon season (May to October) ranging between 140 $\mathrm{mm}$ to $1040 \mathrm{~mm}$. Temperature ranges from $16^{\circ} \mathrm{C}$ to $33^{\circ} \mathrm{C}$, whereas humidity ranges from $29 \%$ to $99 \%$ (BBS 2011).

In Bangladesh an assessment of plant diversity of different national parks and wildlife sanctuary are already being started (Uddin et al. 1998, Uddin and Rahman 1999, Uddin et al. 2011, Uddin and Hassan 2004, 2010, Uddin et al. 2013). So far floristic literature review, no assessment records of angiosperm plant diversity was found for Nijhum Dweep except few plant names in the forest management plan. In the present study an attempt has been made to attain the following objectives: to assess the angiosperm plant diversity, to identify any threats and to suggest some possible conservation measures for the Nijhum Dweep conservation.

\section{Materials and Methods}

Plant sample collections (Hyland 1972, Balick et al. 1982 and Alexiades 1996) from the study area have been done in suitable time of the year of 2013 and 2014 paying three visits. Speciemens were collected from mangrove, meadow, cultivated land, roadside and homestead area. Special efforts were given to find species of conservation concern including threatened, endemic and rare. Voucher specimens processed using standard herbarium techniques (Hyland 1972). The specimens were identified consulting different Floras viz., Hooker 1872-1897, Prain 1903, Uddin and Hassan 2004, Siddiqui et al. 2007c and Ahmed et al. 2008a, 2008b, 2009b, 2009c, 2009d, 2009e. Specimens available at Dhaka University Salar Khan Herbarium (DUSH) were consulted in identifying the collected plant specimens. The updated nomenclature of the species followed Siddiqui et al. 2007c and Ahmed et al. 2008a, 2008b, 2009b, 2009c, 2009d, and 2009e). Voucher specimens are deposited at DUSH. 


\section{Results and Discussion}

A total of 152 plant species under 56 families has been recorded from Nijhum Dweep. For each species local name, scientific name, family, habit and habitat are provided (Table 1). Among the species, 66 are represented by to trees, 15 by shrubs, 58 by herbs and 13 by climbers (Fig. 1). From the recorded species, 51\% species represented by 11 families and $49 \%$ species represented by 45 families (Fig. 2). Fabaceae is the largest family in the Dicotyledon having 10 species whereas Poaceae is the largest family in Monocotyledon having 12 species. We compared plants species recorded in the Nijhum Dweep with medicinal plants data base of Bangladesh. Within recorded plants, 68\% found to be medicinal and 32\% used for other purposes (Fig. 3). Data analysis also showed that homestead supported maximum plants followed by road side, cultivated land, mangrove and mangrove meadow (Fig. 4). Dolichandrone spathacea, a threatened plant species of Bangladesh was recorded from the mangrove forest (Ara et al. 2013). Observations also revealed that occurrence of seven species including Bruguiera gymnorrhiza, Diospyros blancoi, Derris trifoliata, Heliotropium curassavicum, Tamarix gallica, Typha elephentina and Sarcolobus carinatus in the study area might be rare. To confirm such status further detailed survey is needed.

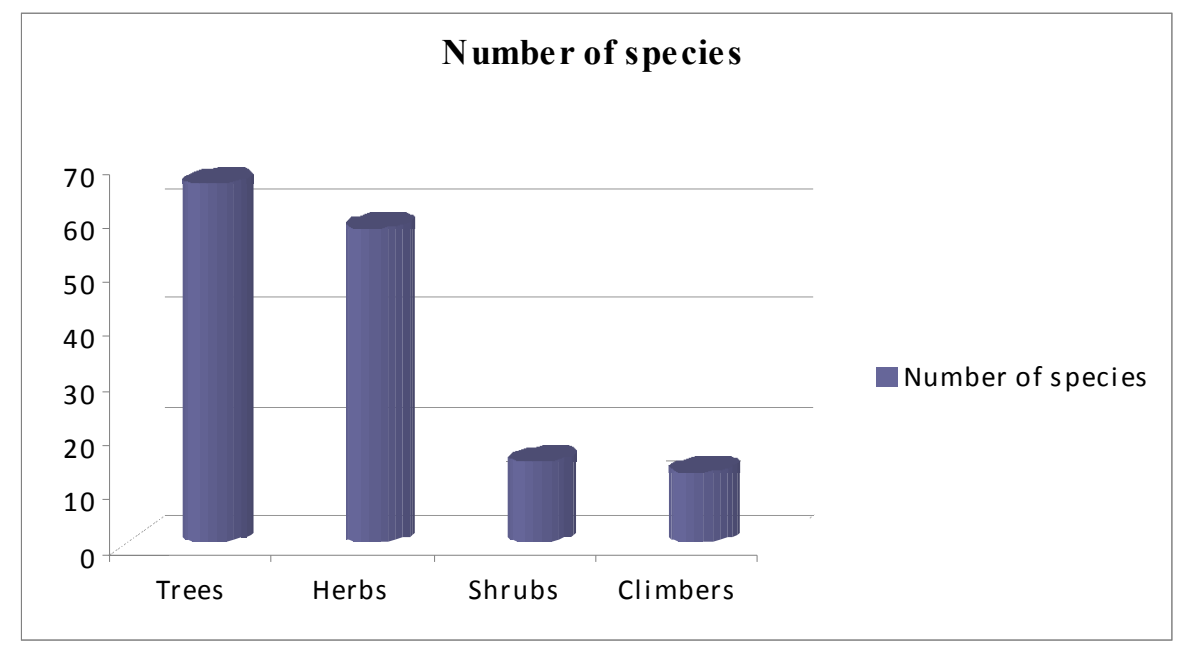

Fig. 1. Different life forms of plant species. 


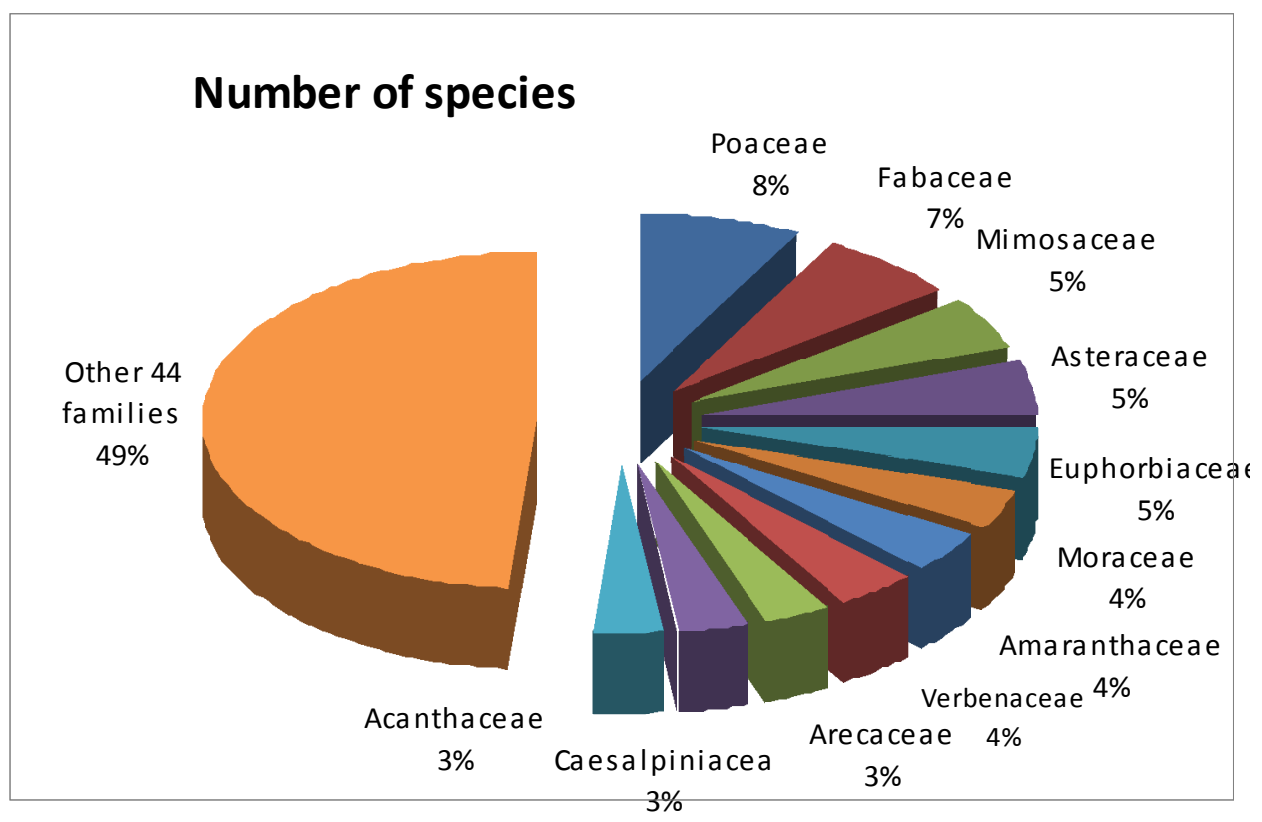

Fig. 2. Distribution of species in the families.

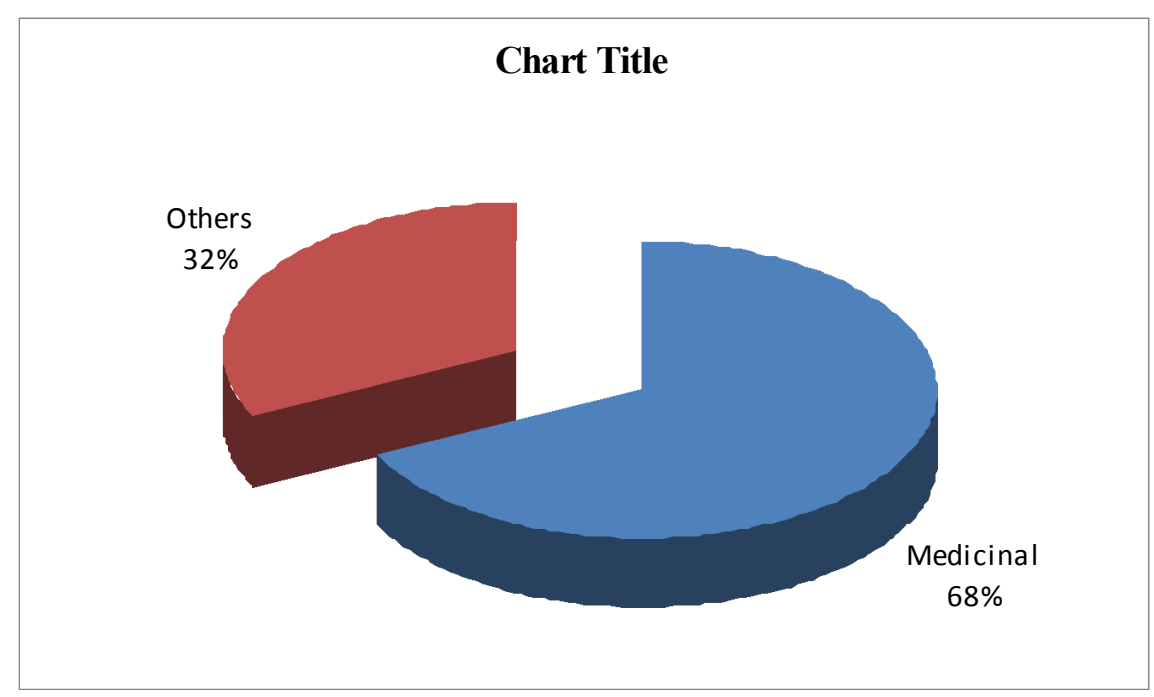

Fig. 3. Relative percentage of medicinal plants. 


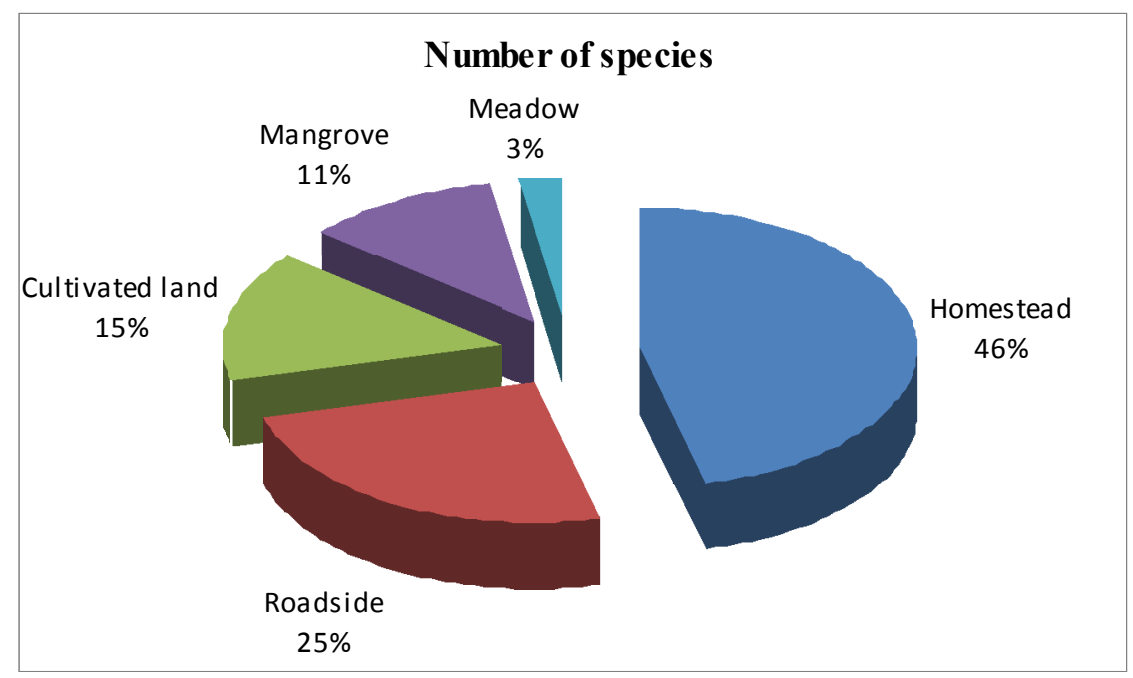

Fig. 4. Percentage of plant species in different habitats.

Observations further revealed that the Island showed different types of land used patterns. Each pattern has been occupied by different types of angiosperm plants. In the inundated area of tidal action, the forest has been formed by mangrove vegetation. Among the vegetation, the most common tree species in the top canopy is Sonneratia apetala (Keora). Associate species in this canopy is Avicennia officinalis (Baine). The middle canopy mainly dominated by only Excoecaria agallocha (Geoa). Two rare tree species also recorded in this layer of vegetation. These are Bruguiera gymnorrhiza (Kakra) and Dolichandrone spathacea. The ground primarily covered by Zoysia matrella, Porteresia coarchtata, Fimbristylis acuminata and Fimbristylis ferruginea.

Table 1. Plant diversity of Nijhum Dweep ( $\mathrm{T}=$ tree, $\mathrm{S}=$ shrub, $\mathrm{H}=$ herb, $\mathrm{C}=$ climber).

\begin{tabular}{|c|c|c|c|c|}
\hline Scientifc name & Local name & Family & Habit & Habitat \\
\hline Acacia nilotica $\mathrm{L}$. & Babla & Mimosaceae & $\mathrm{T}$ & Homestead \\
\hline Acanthus ilicifolius L. & Hargoza & Acanthaceae & $\mathrm{S}$ & Mangrove \\
\hline Adenanthera pavonina $\mathrm{L}$. & Lalchandon & Mimosaceae & $\mathrm{T}$ & Homestead \\
\hline Aegle marmelose (L.) Corr. & Bel & Rutaceae & $\mathrm{T}$ & Homestead \\
\hline Ageratum conyzoides (L.) L. & Fulkuri & Asteraceae & $\mathrm{H}$ & Roadside \\
\hline $\begin{array}{l}\text { Albizia lebbeck (L.) Benth. \& } \\
\text { Hook. }\end{array}$ & Shilkoroi & Mimosaceae & $\mathrm{T}$ & Homestead \\
\hline Albizia procera (Roxb.) Benth. & Sadakoroi & Mimosaceae & $\mathrm{T}$ & Homestead \\
\hline $\begin{array}{l}\text { Albizia richardiana (Voigt.) King } \\
\text { \& Prain. }\end{array}$ & Shiris & Mimosaceae & $\mathrm{T}$ & Homestead \\
\hline Albizia saman (Jacq.) Merr. & Botkoroi & Mimosaceae & $\mathrm{T}$ & Homestead \\
\hline $\begin{array}{l}\text { Alocasia macrorrhizos (L.) G. } \\
\text { Don }\end{array}$ & Mankachu & Araceae & $\mathrm{H}$ & Homestead \\
\hline
\end{tabular}


Contd.

\begin{tabular}{|c|c|c|c|c|}
\hline Scientifc name & Local name & Family & Habit & Habitat \\
\hline $\begin{array}{l}\text { Alternanthera philoxeroides } \\
\text { (Mart.) Griseb. }\end{array}$ & Helencha & Amarnthaceae & $\mathrm{H}$ & $\begin{array}{l}\text { Cultivated } \\
\text { land }\end{array}$ \\
\hline $\begin{array}{l}\text { Alternanthera sessilis (L.) R. Br. } \\
\text { Ex DC.. }\end{array}$ & Hainchashak & Amaranthaceae & $\mathrm{H}$ & $\begin{array}{l}\text { Cultivated } \\
\text { land }\end{array}$ \\
\hline Amaranthus spinosus L. & Kantanote & Amaranthaceae & $\mathrm{H}$ & Roadside \\
\hline Amaranthus viridis $\mathrm{L}$. & data shak & Amaranthaceae & $\mathrm{H}$ & Homestead \\
\hline Annona squamosa $\mathrm{L}$. & Ata & Annonaceae & $\mathrm{T}$ & Homestead \\
\hline $\begin{array}{l}\text { Aphanamixis polystachya (Wall.) } \\
\text { R. N. Parker }\end{array}$ & Pitraj & Meliaceae & $\mathrm{T}$ & Homestead \\
\hline Areca catechu $\mathrm{L}$ & Supari & Arecaceae & $\mathrm{T}$ & Homestead \\
\hline Artocarpus heterophyllus Lamk. & Kathal & Moraceae & $\mathrm{T}$ & Roadside \\
\hline Artocarpus lacucha Buch.-Ham. & Dewa & Moraceae & $\mathrm{T}$ & Homestead \\
\hline Averrhoa carambola $\mathrm{L}$ & Kamranga & Averrhoaceae & $\mathrm{T}$ & Homestead \\
\hline Avicennia officinalis $\mathrm{L}$. & Bain & Verbenaceae & $\mathrm{T}$ & Mangrove \\
\hline $\begin{array}{l}\text { Axonopus compressus (Sw.) P. } \\
\text { Beauv. }\end{array}$ & Dhakagass & Poaceae & $\mathrm{H}$ & Roadside \\
\hline Azadirachta indica A. Juss. & Neem & Meliaceae & $\mathrm{T}$ & Homestead \\
\hline Bacopa monnieri (L.) Pennell & Brammi Shak & Scrophulariaceae & $\mathrm{H}$ & Mangrove \\
\hline Bambusa balcooa Roxb. & Baijja Bans & Poaceae & $\mathrm{T}$ & Homestead \\
\hline Bauhinia purpurea $\mathrm{L}$. & Kanchan & Caesalpiniacea & $\mathrm{T}$ & Homestead \\
\hline Blumea lacera (Burm. f. ) DC. & Kukurmuta & Asteraceae & $\mathrm{H}$ & Roadside \\
\hline Bombax ceiba $\mathrm{L}$. & Shimultula & Bombaeacace & $\mathrm{T}$ & Homestead \\
\hline Borassus flabellifer L. & Tal & Arecaceae & $\mathrm{T}$ & Roadside \\
\hline $\begin{array}{l}\text { Bruguiera gymnorrhiza (L.) } \\
\text { Lamk. }\end{array}$ & Kakra & Rhizophoraceae & $\mathrm{T}$ & Mangrove \\
\hline $\begin{array}{l}\text { Callistemon citrinus (Curtis) } \\
\text { Skeels }\end{array}$ & Bottle Brush & Myrtaceae & $\mathrm{T}$ & Homestead \\
\hline $\begin{array}{l}\text { Calotropis procera (Aiton) } \\
\text { Dryand }\end{array}$ & Akand & Asclepiadaceae & $\mathrm{S}$ & Roadside \\
\hline Capsicum frutescens $\mathrm{L}$. & Morich & Solanaceae & $\mathrm{H}$ & $\begin{array}{l}\text { Cultivated } \\
\text { land }\end{array}$ \\
\hline Carica papaya $\mathrm{L}$. & Pepe & Caricaceae & $\mathrm{S}$ & Homestead \\
\hline Cassia fistula $\mathrm{L}$. & Sonalu & Caesalpiniaceae & $\mathrm{T}$ & Roadside \\
\hline Casuarina equisetifolia L. & Jau & Casuarinaceae & $\mathrm{T}$ & Roadside \\
\hline $\begin{array}{l}\text { Cayratia japonica (Thunb.) } \\
\text { Gagnepain. }\end{array}$ & - & Vitaceae & $\mathrm{C}$ & Roadside \\
\hline Centella asiatica (L.) Urban & Adamoni & Apiaceae & $\mathrm{H}$ & Roadside \\
\hline $\begin{array}{l}\text { Chrysalidocarpus lutescens } \\
\text { (Bory) H. Wendl. }\end{array}$ & Arecapalm & Arecaceae & $\mathrm{T}$ & Homestead \\
\hline $\begin{array}{l}\text { Chrysopogon aciculatus (Retz.) } \\
\text { Trin. }\end{array}$ & Premkanta & Poaceae & $\mathrm{H}$ & Roadside \\
\hline Citrus maxima (Burm. F.) Merr. & Jambura & Rutaceae & $\mathrm{T}$ & Homestead \\
\hline $\begin{array}{l}\text { Clerodendrum indicum (L.) } \\
\text { Kuntze }\end{array}$ & Bhat & Verbenaceae & $\mathrm{S}$ & Mangrove \\
\hline Cocos nucifera $\mathrm{L}$. & Narikel & Arecaceae & $\mathrm{T}$ & Homestead \\
\hline Colocasia esculenta (L.) Schott & Kachu & Araceae & $\mathrm{H}$ & Homestead \\
\hline Crotalaria juncea $\mathrm{L}$. & Junjuni & Fabaceae & $\mathrm{H}$ & Roadside \\
\hline Croton bonplandianus Baill. & Bankhira & Euphorbiaceae & $\mathrm{H}$ & Roadside \\
\hline $\begin{array}{l}\text { Cryptocoryne retrospiralis } \\
\text { (Roxb.) Fisch. }\end{array}$ & Kelakachu & Araceae & $\mathrm{H}$ & Mangrove \\
\hline
\end{tabular}


Contd.

\begin{tabular}{|c|c|c|c|c|}
\hline Scientifc name & Local name & Family & Habit & Habitat \\
\hline Cucurbita maxima Duchesne & Misti kumra & Cucurbitaceae & $\mathrm{C}$ & Homestead \\
\hline Cuscuta reflexa Roxb. & Shwarnalata & Cuscutaceae & $\mathrm{C}$ & Roadside \\
\hline Cyclea barbata Miers. & Patalpur & Menispermaceae & $\mathrm{C}$ & Roadside \\
\hline Cynodon dactylon (L.) Pers. & Durbagass & Poaceae & $\mathrm{H}$ & Homestead \\
\hline Cyperus rotundus $\mathrm{L}$. & Muthagass & Cyperaceae & $\mathrm{H}$ & $\begin{array}{l}\text { Cultivated } \\
\text { land }\end{array}$ \\
\hline Dalbergia sissoo DC. & Shissu & Fabaceae & $\mathrm{T}$ & Roadside \\
\hline Delonix regia Rafin. & Krishnachura & Caesalpiniaceae & $\mathrm{T}$ & Roadside \\
\hline $\begin{array}{l}\text { Dentella repens (L.) J. R. \& G. } \\
\text { Forst. }\end{array}$ & Bhuipat & Rubiaceae & $\mathrm{H}$ & $\begin{array}{l}\text { Cultivated } \\
\text { land }\end{array}$ \\
\hline Derris scandens (Roxb.) Benth. & Kalilata & Fabaceae & $\mathrm{C}$ & Mangrove \\
\hline Derris trifoliata Lour. & Kalilota & Fabaceae & $\mathrm{C}$ & Mangrove \\
\hline Dioscorea bulbifera L. & Matialu & Dioscoriaceae & $\mathrm{C}$ & Homestead \\
\hline Diospyros blancoi A. DC. & Bilatigab & Ebenaceae & $\mathrm{T}$ & Homestead \\
\hline $\begin{array}{l}\text { Diospyros malabarica (Desr.) } \\
\text { Kostel. }\end{array}$ & Deshigab & Ebenaceae & $\mathrm{T}$ & Homestead \\
\hline $\begin{array}{l}\text { Dolichandrone spathacea (L.f.) } \\
\text { K. Schum. }\end{array}$ & Chamhechandan & Bignoniaceae & $\mathrm{T}$ & Mangrove \\
\hline Eclipta prostrata (L.) Mant. & Keshoraj & Asteraceae & $\mathrm{H}$ & $\begin{array}{l}\text { Cultivated } \\
\text { land }\end{array}$ \\
\hline $\begin{array}{l}\text { Eichhornia crassipes (Mart.) } \\
\text { Solms }\end{array}$ & Kachripana & Pontederiaceae & $\mathrm{H}$ & Homestead \\
\hline $\begin{array}{l}\text { Elaeocarpus tectorius (Lour.) } \\
\text { Poir. }\end{array}$ & Jolpai & Elaeocarpaceae & $\mathrm{T}$ & $\begin{array}{l}\text { Cultivated } \\
\text { land }\end{array}$ \\
\hline $\begin{array}{l}\text { Eleocharis geniculata (L.) Roem. } \\
\text { \& Schult. }\end{array}$ & Joraghasi & Cyperaceae & $\mathrm{H}$ & $\begin{array}{l}\text { Cultivated } \\
\text { land }\end{array}$ \\
\hline Eleusine indica (L.) Gaertn. & Malankuri & Poaceae & $\mathrm{H}$ & $\begin{array}{l}\text { Cultivated } \\
\text { land }\end{array}$ \\
\hline Erythrina indica Lamk. & Painnamandar & Fabaceae & $\mathrm{T}$ & Homestead \\
\hline Erythrina ovalifolia Roxb. & Mandar & Fabaceae & $\mathrm{T}$ & Homestead \\
\hline Excoecaria agallocha $\mathrm{L}$. & Gewa & Euphorbiaceae & $\mathrm{T}$ & Mangrove \\
\hline Ficus benghalensis L. & Bot & Moraceae & $\mathrm{T}$ & Roadside \\
\hline Ficus hispida L. f. & Dumur & Moraceae & $\mathrm{T}$ & Homestead \\
\hline Ficus infectoria Roxb. & Pakur & Moraceae & $\mathrm{T}$ & Roadside \\
\hline Ficus racemosa L. & Jogdumur & Moraceae & $\mathrm{T}$ & Roadside \\
\hline Fimbristylis acuminata Vahl & - & Cyperaceae & $\mathrm{H}$ & $\begin{array}{l}\text { Cultivated } \\
\text { land }\end{array}$ \\
\hline Fimbristylis ferruginea (L.) Vahl & - & Cyperaceae & $\mathrm{H}$ & $\begin{array}{l}\text { Cultivated } \\
\text { land }\end{array}$ \\
\hline Garcinia cowa Roxb. ex DC. & Kao & Clusiaceae & $\mathrm{T}$ & Homestead \\
\hline Gardenia jasminoides J.Ellis & Gandhraj & Rubiaceae & $\mathrm{S}$ & Homestead \\
\hline Gomphrena globosa L. & Botamphul & Amaranthaceae & $\mathrm{H}$ & Roadside \\
\hline $\begin{array}{l}\text { Grangea maderaspatana (L.) } \\
\text { Poir. }\end{array}$ & Nemuti & Asteraceae & $\mathrm{H}$ & $\begin{array}{l}\text { Cultivated } \\
\text { land }\end{array}$ \\
\hline Heliotropium curassavicum $\mathrm{L}$. & Nuinna & Boragnaceae & $\mathrm{H}$ & $\begin{array}{l}\text { Cultivated } \\
\text { land }\end{array}$ \\
\hline Heliotropium indicum $\mathrm{L}$. & Hatisur & Boragnaceae & $\mathrm{H}$ & $\begin{array}{l}\text { Cultivated } \\
\text { land }\end{array}$ \\
\hline Hibiscus rosa-sinensis L. & Joba & Malvaceae & $\mathrm{S}$ & Homestead \\
\hline
\end{tabular}


Contd.

\begin{tabular}{|c|c|c|c|c|}
\hline Scientife name & Local name & Family & Habit & Habitat \\
\hline Hygrophila phlomoides Nees & - & Acanthaceae & $\mathrm{H}$ & $\begin{array}{l}\text { Cultivated } \\
\text { land }\end{array}$ \\
\hline $\begin{array}{l}\text { Hygrophila salicifolia (Vahl) } \\
\text { Nees }\end{array}$ & Kakmasha & Acanthaceae & $\mathrm{H}$ & $\begin{array}{l}\text { Cultivated } \\
\text { land }\end{array}$ \\
\hline Ipomoea aquatica Forssk. & Kolmi & Convolvulaceae & $\mathrm{H}$ & Homestead \\
\hline $\begin{array}{l}\text { Ipomoea fistulosa Mart. ex } \\
\text { Choisy }\end{array}$ & Dolkolmi & Convolvulaceae & $\mathrm{H}$ & Roadside \\
\hline Ipomoea pes-caprae (L.) R. Br. & Chagalkhuri & Convolvulaceae & $\mathrm{H}$ & $\begin{array}{l}\text { Cultivated } \\
\text { land }\end{array}$ \\
\hline Lablab purpurea (L.) Sweet & Seem & Fabaceae & $\mathrm{C}$ & Homestead \\
\hline $\begin{array}{l}\text { Lagenaria siceraria (Molina) } \\
\text { Standl. }\end{array}$ & Lao & Cucurbitaceae & $\mathrm{C}$ & Homestead \\
\hline Lagerstroemia indica $\mathrm{L}$. & Cheri & Lythraceae & $\mathrm{T}$ & Homestead \\
\hline Lagerstroemia speciosa (L.) Pers. & Jarul & Lythraceae & $\mathrm{T}$ & Homestead \\
\hline $\begin{array}{l}\text { Lannea coromandelica (Houtt.) } \\
\text { Merr. }\end{array}$ & Bhadi & Anacardiaceae & $\mathrm{T}$ & Homestead \\
\hline Lawsonia inermis L. & Mehendi & Lythraceae & $\mathrm{T}$ & Homestead \\
\hline $\begin{array}{l}\text { Leucaena leucocephala (Lamk.) } \\
\text { de Wit. }\end{array}$ & Epilepil & Mimosaceae & $\mathrm{T}$ & Roadside \\
\hline $\begin{array}{l}\text { Lippia alba (Mill.) N. E. Br. Ex } \\
\text { Britt. \&Wilson }\end{array}$ & Bhuiokra & Verbenaceae & $\mathrm{H}$ & Roadside \\
\hline Litchi chinensis Sonn. & Lichu & Sapindaceae & $\mathrm{T}$ & Homestead \\
\hline Mangifera indica $\mathrm{L}$. & Aam & Anacardiaceae & $\mathrm{T}$ & Homestead \\
\hline Melia azederach L. & Goraneem & Meliaceae & $\mathrm{T}$ & Homestead \\
\hline Mikania micratha Kunth & Assamilata & Asteraceae & $\mathrm{C}$ & Roadside \\
\hline Morinda citrifolia $\mathrm{L}$. & Banach & Rubiaceae & $\mathrm{S}$ & Homestead \\
\hline Moringa oleifera Lamk. & Shajna & Moringaceae & $\mathrm{T}$ & Homestead \\
\hline $\begin{array}{l}\text { Mosla dainthera (Buch.-Ham. ex } \\
\text { Roxb.) Maxim. }\end{array}$ & - & Lamiaceae & $\mathrm{H}$ & Homestead \\
\hline Mucuna gigantean (Willd.) DC. & Bara-alkuchi & Fabaceae & $\mathrm{C}$ & Roadside \\
\hline Musa paradisiaca $\mathrm{L}$. & Kola & Musaceae & $\mathrm{H}$ & Homestead \\
\hline $\begin{array}{l}\text { Nelsonia canescens (Lamk.) } \\
\text { Spreng. }\end{array}$ & - & Acanthaceae & $\mathrm{H}$ & Roadside \\
\hline Ocimum sanctum $\mathrm{L}$. & Tulsi & Lamiaceae & $\mathrm{H}$ & Homestead \\
\hline Paspalum distichum $\mathrm{L}$. & Gitlaghas & Poaceae & $\mathrm{H}$ & $\begin{array}{l}\text { Cultivated } \\
\text { land }\end{array}$ \\
\hline Paspalum vaginatum $\mathrm{Sw}$. & - & Poaceae & $\mathrm{H}$ & Meadow \\
\hline $\begin{array}{l}\text { Phaulopsis imbricata (Forssk.) } \\
\text { Sweet }\end{array}$ & Kantasi & Acanthaceae & $\mathrm{H}$ & Roadside \\
\hline Phoenix sylvestris (L.) Roxb. & Khejur & Arecaceae & $\mathrm{T}$ & Roadside \\
\hline Phyla nodiflora (L.) Greene & Kanghas & Verbenaceae & $\mathrm{H}$ & $\begin{array}{l}\text { Cultivated } \\
\text { land }\end{array}$ \\
\hline $\begin{array}{l}\text { Phyllanthus acidus (L.) Skeels } \\
\text { Phyllanthus reticulatus Poir. }\end{array}$ & $\begin{array}{l}\text { Orbori } \\
\text { Sitki }\end{array}$ & $\begin{array}{l}\text { Euphorbiaceae } \\
\text { Euphorbiaceae }\end{array}$ & $\begin{array}{l}\mathrm{T} \\
\mathrm{S}\end{array}$ & $\begin{array}{l}\text { Homestead } \\
\text { Roadside }\end{array}$ \\
\hline $\begin{array}{l}\text { Pithecellobium dulce (Roxb.) } \\
\text { Benth. }\end{array}$ & Khoibabla & Mimosaceae & $\mathrm{T}$ & Homestead \\
\hline Polygonum blebeium R. Br. & - & Polygonaceae & $\mathrm{H}$ & $\begin{array}{l}\text { Cultivated } \\
\text { land }\end{array}$ \\
\hline Pongamia pinnata (L.) Pierre & Koroj & Caesalpiniaceae & $\mathrm{T}$ & Homestead \\
\hline
\end{tabular}


Contd.

\begin{tabular}{|c|c|c|c|c|}
\hline Scientifc name & Local name & Family & Habit & Habitat \\
\hline $\begin{array}{l}\text { Porteresia coarctata (Roxb.) } \\
\text { Takeoka }\end{array}$ & Uri & Poaceae & $\mathrm{H}$ & Meadow \\
\hline Portulaca oleracea L. & Nuainashak & Potulacaceae & $\mathrm{H}$ & $\begin{array}{l}\text { Cultivated } \\
\text { land }\end{array}$ \\
\hline $\begin{array}{l}\text { Psilotrichum ferrugineum (Roxb.) } \\
\text { Moq.-Tand. }\end{array}$ & Putishak & Amarnthaceae & $\mathrm{H}$ & $\begin{array}{l}\text { Cultivated } \\
\text { land }\end{array}$ \\
\hline Punica granatum $\mathrm{L}$. & Dalim & Punicaceae & $\mathrm{S}$ & Homestead \\
\hline Ricinus communis $\mathrm{L}$. & Keron & Euphorbiaceae & $\mathrm{S}$ & Homestead \\
\hline Saccharum spontaneum L. & Chan & Poaceae & $\mathrm{H}$ & Mangrove \\
\hline Sapium indicum Willd. & Harua & Euphorbiaceae & $\mathrm{T}$ & Roadside \\
\hline Sarcolobus carinatus Wall. & - & Asclepiadaceae & $\mathrm{C}$ & Mangrove \\
\hline $\begin{array}{l}\text { Schumannianthus dichotomus } \\
\text { (Roxb.) Ganep. }\end{array}$ & Patipata & Meratnaceae & $\mathrm{H}$ & Homestead \\
\hline Sesbania grandiflora (L.) Pers. & Bakul ful & Fabaceae & $\mathrm{S}$ & Homestead \\
\hline Solanum nigrum $\mathrm{L}$. & Titbegun & Solanaceae & $\mathrm{H}$ & Roadside \\
\hline Sonneratia apetala Buch.-Ham. & Keora & Sonneratiaceae & $\mathrm{T}$ & Mangrove \\
\hline Spondias pinnata (L. f.) Kurz. & Deshi amra & Anacardiaceae & $\mathrm{T}$ & Homestead \\
\hline $\begin{array}{l}\text { Stephania japonica (Thunb.) } \\
\text { Miers }\end{array}$ & Muchchanilata & Menispermaceae & $\mathrm{C}$ & Roadside \\
\hline Swietenia mahagoni (L.) Jacq. & Mehagoni & Meliaceae & $\mathrm{T}$ & Homestead \\
\hline Syzygium cumini (L.) Skeels & Kalojam & Myrtaceae & $\mathrm{T}$ & Homestead \\
\hline Syzygium fruticosum (Roxb.) DC. & Bhutijam & Myrtaceae & $\mathrm{T}$ & Homestead \\
\hline $\begin{array}{l}\text { Syzygium malaccense (L.) Merr. } \\
\text { \& L. M. Perry }\end{array}$ & Jamrul & Myrtaceae & $\mathrm{T}$ & Homestead \\
\hline Tamarindus indica $\mathrm{L}$. & Tentul & Caesalpiniaceae & $\mathrm{T}$ & Homestead \\
\hline Tamarix gallica $\mathrm{L}$. & Nonajau & Tamaricaceae & $\mathrm{S}$ & Mangrove \\
\hline $\begin{array}{l}\text { Terminalia arjuna (Roxb. ex DC.) } \\
\text { Wight \& Arn. }\end{array}$ & Arjun & Combretaceae & $\mathrm{T}$ & Roadside \\
\hline Terminalia catappa $\mathrm{L}$. & Katgolap & Combretaceae & $\mathrm{T}$ & Homestead \\
\hline $\begin{array}{l}\text { Terminalia chebula } \\
\text { (Gaertn.)Retz. }\end{array}$ & Bohera & Combretaceae & $\mathrm{T}$ & Homestead \\
\hline $\begin{array}{l}\text { Thespesia populnea (L.) Sol. Ex } \\
\text { Corr. }\end{array}$ & Paresh & Malvaceae & S & Mangrove \\
\hline Trewia nudiflora $\mathrm{L}$. & Pidali & Euphorbiaceae & $\mathrm{T}$ & Homestead \\
\hline Typha elephantina Roxb. & Hogla & Typhaceae & $\mathrm{H}$ & $\begin{array}{l}\text { Cultivated } \\
\text { land }\end{array}$ \\
\hline Urena lobata $\mathrm{L}$ & Jogagota & Malvaceae & $\mathrm{H}$ & Homestead \\
\hline Vernonia cinerea (L.) Less. & Kuksim & Asteraceae & $\mathrm{H}$ & Roadside \\
\hline Vitex negundo L.. & Nishinda & Verbnaceae & $\mathrm{S}$ & Roadside \\
\hline Vitex trifolia $\mathrm{L} . . \mathrm{f}$. & Neelnishinda & Verbenaceae & $\mathrm{S}$ & Roadside \\
\hline Wedelia calendulacea (L.) Less. & Mohabingaraj & Asteraceae & $\mathrm{H}$ & Mangrove \\
\hline $\begin{array}{l}\text { Xanthium indicum Koen. ex } \\
\text { Roxb. }\end{array}$ & Ghagrashak & Asteraceae & $\mathrm{H}$ & $\begin{array}{l}\text { Cultivated } \\
\text { land }\end{array}$ \\
\hline Xanthosoma violaceum Schott & Dudkachu & Araceae & $\mathrm{H}$ & Homestead \\
\hline Ziziphus mauritiana Lamk. & Boroi & Rhamnaceae & $\mathrm{T}$ & Homestead \\
\hline Zoysia matrella (L.) Merr. & Gass & Poaceae & $\mathrm{H}$ & Meadow \\
\hline Zoysia tenuifolia Willd. ex Thiele & - & Poaceae & $\mathrm{H}$ & Meadow \\
\hline
\end{tabular}


Near the bank of channel and water the dominant vegetations are Acanthus ilicifolious, Porteresia coarchtata, Cryptocoryne retrospiralis, Saccharum sp., Wedelia calendula, Baccopa monnieri, Phylla nodiflora, Hibiscus poplena, Dalbergis spinosa, Zoysia matrella and Clerodendrun indicum. The main climbers of the area are Derris scandens, Derris trifoliata and the rare occurrence Sarcolobus carinatus. A rare occurrence of Tamarix gallica (Nuinna Jao) also recorded in the Mangrove area.

The meadow near the river is dominated by Porteresia coarchtata, Zoysia matrella and Paspalum vaginatum. Sporadic distribution of seedling of Excoecaria agallocha tree was also recorded in the meadow area. Once wildlife particularly spotted deer used to browse and roam in such meadow area. Unfortunately this area now converted into domestic buffalos grazing field. The cultivated land mainly is used for rice production during rainy season. In the summer the land covered by a number herbaceous plants. Among them the common species are Bacopa monnieri, Dentella repens, Psilotrichum ferrugineum, Polygonum plebejum, Phyla nodiflora, Grangea madarspatana, Xanthium indicum, Portulaca oleracea, Heliotropium curassavicum, H. indicum, Eclipta prostrata and Alternanthera sessilis.. A rare occurrence of Typha elephentanea (Hogla) also recorded in the wetland. Some summer crops including chili, watermelon, sweet pumpkin, sweet potato, Tomato, legumes etc. were observed to cultivate in this area. The vegetation of the homestead is very rich with so many planted species like main land homestead vegetation. The most common species are Rain tree (Albizia saman), Mango (Mangifera indica), Jackfruit (Artocarpus heterophyllus), Coconut (Cocos nucifera), Areca palm (Areaca catechu), Date palm (Phoenix sylvestris), Talipalm (Borassus flabellifer), Koroi (Albizia procera) and Shilkoroi (Albizia labbeck). Both sides of the road always support dense vegetation of different life forms of plant species. The most common tree species in the road sides are Date palm (Phoenix sylvestris), Talipalm (Borassus flabellifer), Akanda (Calotropis procera), Pidali (Trewia nudiflora), Rain tree (Samanea saman), Jao (Casuarina litoralis), Sonalu (Cassia fistula), Arjun (Terminalia arjuna), and Epilepil (Leucaena leucocephala). A good number of shrubs, herbs and climbers also colonized in both sides of the road.

For the management of the Island, threats determination is very important challenge. Without such work management plan could not be worked properly to achieve desired goal. In our study, discussion with foresters, interviews with local people, group discussion and observation in the field, a number of threats to plant diversity in Nijhum Dweep have been identified. 


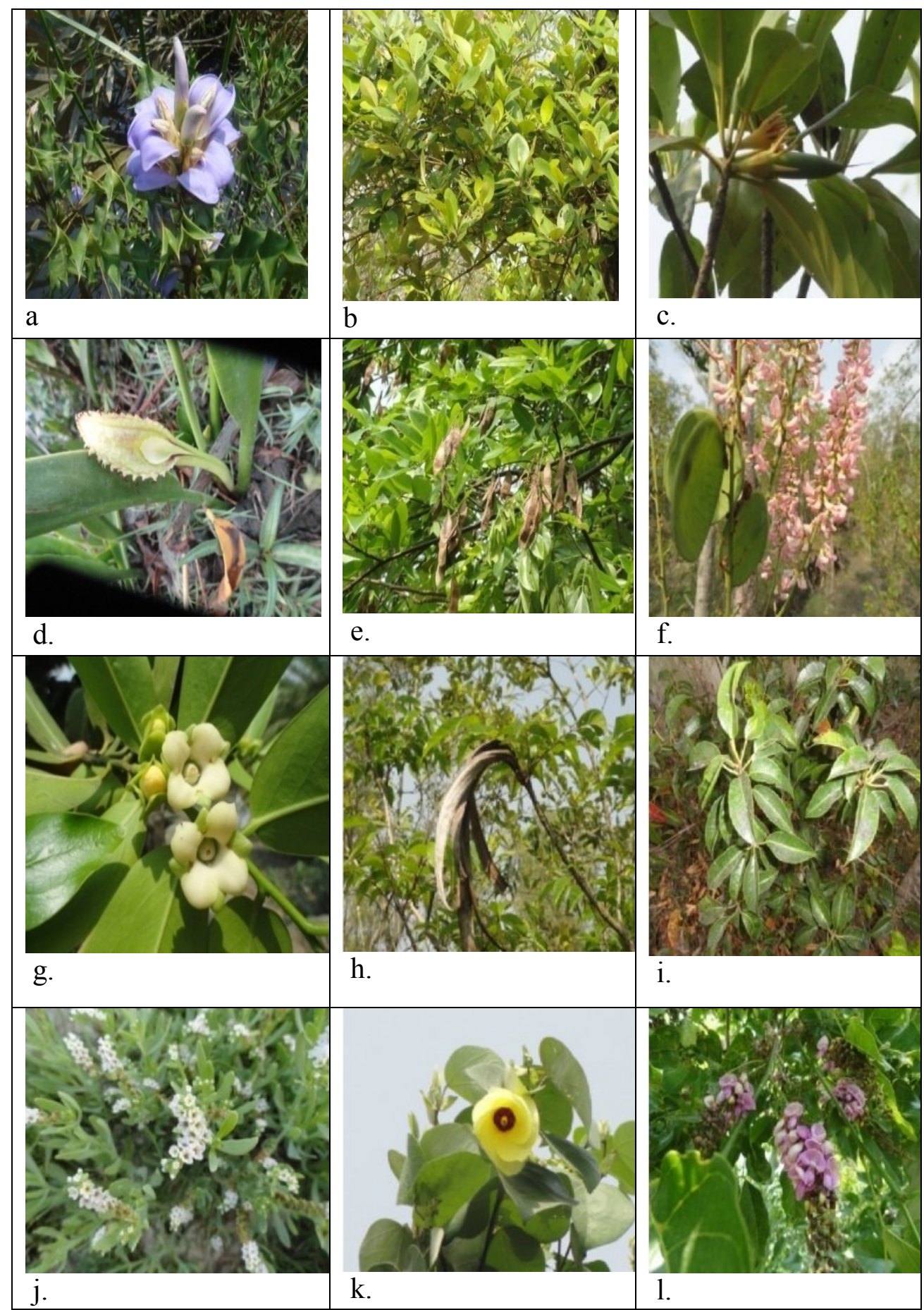




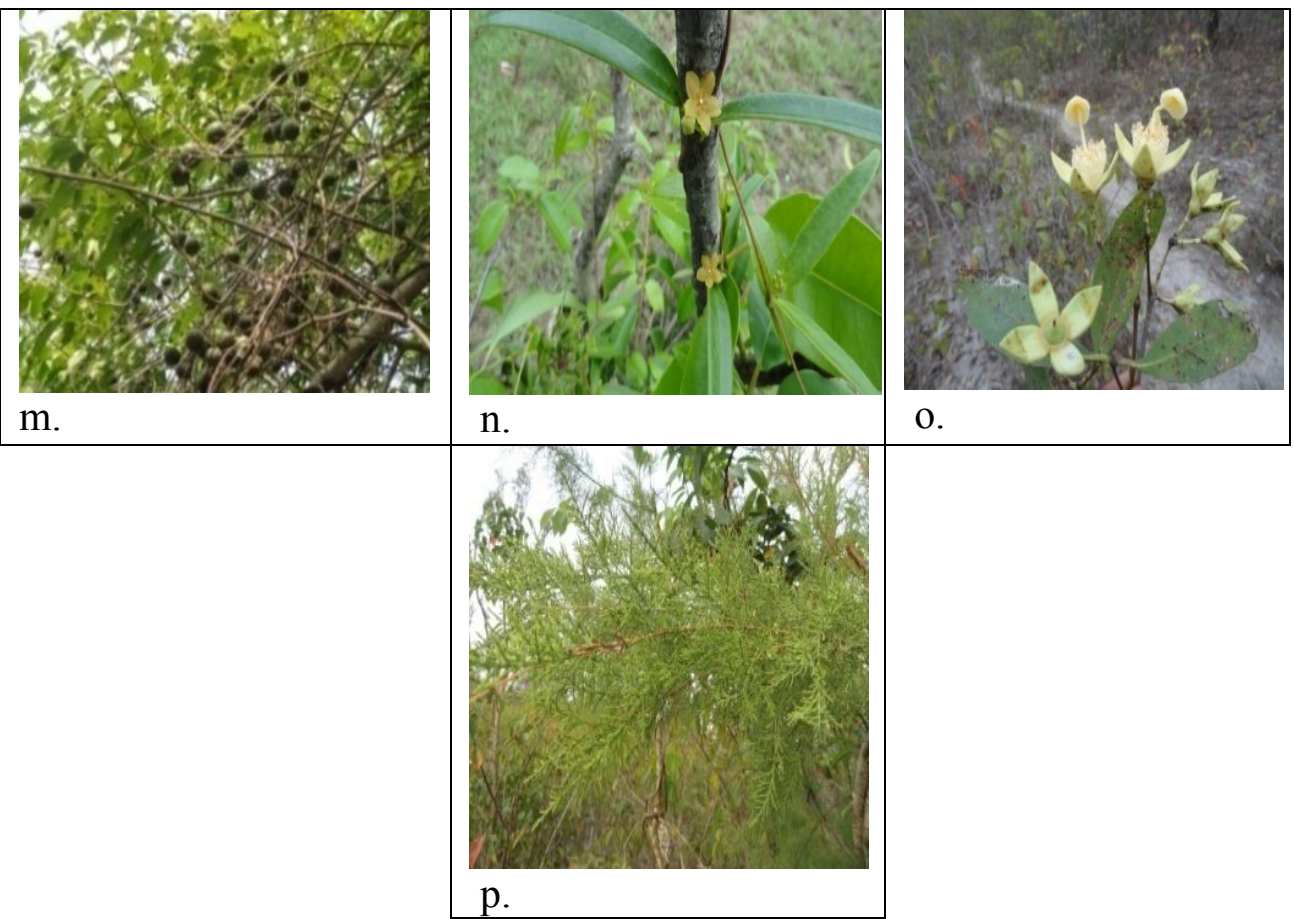

Plate 1: Some salt tolerant plants of the Island a. Acanthus ilicifolius b. Avicennia officinalis c. Bruguiera gymnorrhiza d. Cryptocoryne retrospiralis e. Derris scandens f. Derris trifoliata g. Diospyros blancoi h. Dolichandrone spathacea i. Excoecaria agallocha j. Heliotropium curassavicum k. Hibiscus populnea 1. Pongamia pinnata m. Sapium indicum n. Sarcolobus carinatus o. Sonneratia apetala p. Tamarix gallica.

These are uncontrolled population pressure, human settlement, encroachment into the forested areas, increasing rate of land value, land lease by District commissioner, illegal logging, overgrazing by domestic buffalos, regeneration problem in canopy trees, edge effect, lack of inundation, keora plant in extinction process in some area, lack of awareness about the value of biodiversity, lack of manpower in the forest department, and political influence in encroachment into the forest area.

Based on our present survey results and observations, a number of possible measures have been suggested for the future management plan of Nijhum Dweep. These are provided below: Sharp boundary can be created around the national park using plantation of native tree species. Ownership conflict between District Commissioner (DC) and forest department should be solved and also ensuring ownership of newly accreted land. Grazing in both forest area and meadow land by domestic animals should be controlled. The study area could be used as climate change monitoring area/biodiversity conservation monitoring area. Plantation program within the Island could be taken to promote 
biodiversity particularly deer and birds population. Capacity of local forest department should be doubled. Awareness program should be conducted to provide the opportunity to the local people about the impact of overpopulation, illegal logging and climate change. Local stakeholder should be involved in the management process of the study area. Guideline could be written for ecotourists to watch biodiversity.

The present list of angiosperm plant diversity (152 species) is still considered as preliminary for the Nijhum Dweep. There might be some more species yet to be listed and few specimens remain unidentified. Based on the field observations and present preliminary results it may be concluded that the Island is not so rich in the angiosperm plant diversity and the area is the home for one threatened plant species (Dolichandrone spathacea). Bruguiera gymnorrhiza, Diospyros blancoi, Derris trifoliata, Heliotropium curassavicum, Tamarix gallica, Typha elephentanea and Sarcolobus carinatus are also found to be rare in the study area. Field observation also confirmed that regeneration of Keora (Sonneratia apetala) and Bain (Avicennia officinalis) species in the habitat are severely hampered because of anthropogenic pressure and over-grazing by domestic animals. Invasive species such as Croton bonplandianus, Urena lobata, Mikania micrantha are another challenge to native species diversity in the study areas. The study suggests for further long term research to focus all aspects of angiosperm plant diversity to help in making proper management plan for this Island. If managed properly, the Nijhum Dweep could be fascinating to tourist and also good sources of revenue for the country. Local community will also be benefited from the Island.

\section{Acknowledgement}

Bangladesh Forest Department and local forest office of Noakhali and Nijhum Dweep are greatly acknowledged for financial support and facilities. The authors are also thankful to the wildlife rescue center of Jahangirnagar University for implementing research work.

\section{References}

Ahmed, Z.U., Begum, Z.N.T., Hassan, M.A., Khondker, M., Kabir, S.M.H., Ahmad, M., Ahmed, A.T.A., Rahman, A.K.A. and Haque, E.U. (Eds) 2008a. Encyclopedia of Flora and Fauna of Bangladesh, Vol. 6. Angiosperms: Dicotyledons (Acanthaceae - Asteraceae). Asiatic Society of Bangladesh, Dhaka, pp. 1-408.

Ahmed, Z.U., Hassan, M.A., Begum, Z.N.T., Khondker, M., Kabir, S.M.H., Ahmad, M., Ahmed, A.T.A., Rahman, A.K.A. and Haque, E.U. (Eds) 2008b. Encyclopedia of Flora and Fauna of Bangladesh, Vol. 12. Angiosperms: Monocotyledons (Orchidaceae Zingiberaceae). Asiatic Society of Bangladesh, Dhaka, pp. 1-552.

Ahmed, Z.U., Hassan, M.A., Begum, Z.N.T., Khondker, M., Kabir, S.M.H., Ahmad, M., Ahmed, A.T.A., Rahman, A.K.A. and Haque, E.U. (Eds) 2009b. Encyclopedia of Flora and Fauna of Bangladesh, Vol. 7. Angiosperms: Dicotyledons (Balsaminaceae Euphorbiaceae). Asiatic Society of Bangladesh, Dhaka, pp. 1-546. 
Ahmed, Z.U., Hassan, M.A., Begum, Z.N.T., Khondker, M., Kabir, S.M.H., Ahmad, M., Ahmed, A.T.A., Rahman, A.K.A. and Haque, E.U. (Eds) 2009c. Encyclopedia of Flora and Fauna of Bangladesh, Vol. 8. Angiosperms: Dicotyledons (Fabaceae - Lythraceae). Asiatic Society of Bangladesh, Dhaka, pp. 1-478.

Ahmed, Z.U., Hassan, M.A., Begum, Z.N.T., Khondker, M., Kabir, S.M.H., Ahmad, M. and Ahmed, A.T.A. (Eds) 2009d. Encyclopedia of Flora and Fauna of Bangladesh, Vol. 9. Angiosperms: Dicotyledons (Magnoliaceae - Punicaceae). Asiatic Society of Bangladesh, Dhaka, pp. 1-488.

Ahmed, Z.U., Hassan, M.A., Begum, Z.N.T., Khondker, M., Kabir, S.M.H., Ahmad, M., and Ahmed, A.T.A. (Eds) 2009e. Encyclopedia of Flora and Fauna of Bangladesh, Vol. 10. Angiosperms: Dicotyledons (Ranunculaceae - Zygophyllaceae). Asiatic Society of Bangladesh, Dhaka, pp. 1-580.

Alexiades, M. N. (ed.). 1996. Selected Guidelines for Ethno botanical Research: A Field Manual. The New York Botanical Garden, New York.

Ara, H., B. Khan and S. N. Uddin. 2013 (eds.) Red data book of vascular plants of Bangladesh, Volume 2. Bangladesh National Herbarium, Dhaka, Bangladesh. 280pp.

Balick, M. J., A. B. Anderson and M. F. da Silva. 1982. Plant taxonomy in Brazilian Amazonia: The state of systematic collection in regional herbaria. Brittonia 14: 463-477.

BBS (Bangladesh Bureau of Statistics) 2011. Monthly Statistical Bulletin, December 2011. Statistics Division, Ministry of Planning, Government of the People's Republic of Bangladesh.

Hooker, J.D. 1872-1897. The flora of British India. Vols. 1-7 London.

Hyland, B.P.M. 1972. A technique for collecting botanical specimens in rain forest. Flora Malesiana Bulletin, 26: 2038-2040.

Prain, D. 1903. Bengal Plants Vol. 1-2: 1-1013pp. First Indian Reprint 1963, Bishen Singh Mahendra Pal Singh Dehra Dun.

Siddiqui, K.U., Islam, M.A., Ahmed, Z.U., Begum, Z.N.T., Hassan, M.A., Khondker, M., Rahman, M.M., Kabir, S.M.H., Ahmad, M., Ahmed, A.T.A., Rahman, A.K.A. and Haque, E.U. (Eds) 2007c. Encyclopedia of Flora and Fauna of Bangladesh, Vol. 11. Angiosperms: Monocotyledons (Agavaceae -Najadaceae). Asiatic Society of Bangladesh, Dhaka, pp. 1399.

Uddin M Z, M. F. Alam, A. S. M. Rahman and M. A. Hassan. 2011. Plant Biodiversity of Fashiakhali Wildlife Santuary, Bangladesh. Accepted for publication in First Bangladesh Forestry Congress Proceeding 2011.

Uddin S.B. and M. A. Rahman. 1999. Angiospermic flora of Himchari National Park, Cox's Bazar, Bangladesh. J. Plant Taxon. 6(1): 31-68.

Uddin S.N., M. S. Khan, M. A. Hassan, and M. K.Alam. M.K. 1998. An annotated checklist of angiospermic flora of Sita Pahar at Kaptai in Bangladesh. Bangladesh J. Plant Taxon. 5(1): 13-46.

Uddin, M. Z. and M. A. Hassan . 2010. Angiosperm diversity of Lawachara National Park (Bangladesh): a preliminary assessment. Bangladesh J. Plant Taxon. 17 (1): 9-22.

Uddin, M. Z. and M. A. Hassan. M.A. 2004. Flora of Rema-Kalenga Wildlife Sanctuary. IUCN Bangladesh Country Office, Dhaka, Bangladesh, vi+120 pp.

Uddin, M.Z., M. F.Alam, M. A. Rahman, and M. A. Hassan. 2013. Diversity in angiosperm flora of Teknaf Wildlife Sanctuary, Bangladesh. Bangladesh J. Plant Taxon. 20(2): 145-162. 